\title{
Experiments with Dynamic Multiplexing and UPC Renegotiation for Video over ATM
}

\author{
Maria Teresa Andrade and Artur Pimenta Alves \\ INESC Porto, Praça da República, 93 R/C, Porto, Portugal \\ FEUP, DEEC, Rua dos Bragas, Porto, Portugal \\ telephone: +351222094238 fax number: +351 222084172 \\ \{mandrade, palves\}@inescn.pt \\ http://telecom.inescn.pt
}

\begin{abstract}
In this paper we present an experimental approach for QoSaware and bandwidth-efficient transmission of multimedia sources over ATM networks. VBR video sources are statistically multiplexed at the application level and the UPC parameters of a single connection for the aggregate traffic are dynamically renegotiated with the network during the session lifetime. A statistical multiplexer computes the required bandwidth for the aggregate traffic and dynamically assigns bandwidth according to the performances requested and network resources availability. A dynamic UPC manager using feedback information sent by the network, may initiate a renegotiation of traffic parameters upon receiving a request from the statistical multiplexer. Performing the connection admission control procedures for the aggregate traffic, allows significant reductions in the value of the total peak rate when compared to the sum of peak rates of individual connections. It also reduces the burstiness of the flow submitted to the network, thus increasing the lifetime of each set of UPC parameters.
\end{abstract}

Keywords: Quality of Service, ATM, UPC, statistical multiplexing, renegotiation

Abbreviations: AAL, ATM Adaptation Layer; ABR, Available Bit Rate; ACTS, Advanced Communications, Technologies \& Services; ATM, Asynchronous Transfer Mode; CAC, Connection Admission Control; CBR, Constant Bit Rate; CDV, Cell Delay Variation; CDVT, Cell Delay Variation Tolerance; CTD, Cell Transfer Delay; EFCI, Explicit Forward Congestion Indication; GOP, Group Of Pictures; MBS, Maximum Burst Size; MPEG, Motion Picture Expert Group; NIC, Network Interface Card; PCR, Peak Cell Rate; MCR, Minimum Cell Rate; QoS, Quality of Service; RM, Resource and Management; SCR, Sustainable Cell Rate; UNI, User Network Interface; UPC, Usage Parameter Control; VBR, Variable Bit Rate; VC, Virtual Circuit; VoD, Video on Demand. 


\section{Introduction}

ATM and MPEG2 are key technologies for the deployment of high-quality networked video services in the present multimedia communications scenario. The use of MPEG2 allows economies in transmission bandwidth while providing a high-quality service. The video compression algorithm reduces the number of bits necessary to represent the video sequences by exploiting spatial and temporal redundancy. However, because redundancy of sequences is variable, constant quality is only obtained if this number of bits is not restricted to a given value.

Transmission of constant quality compressed video streams and therefore variable bit rate streams, is possible in broadband networks using the Asynchronous Transfer Mode. Traditional transmission channels offer only constant bit rate connections which either leads to an inefficient bandwidth utilisation or to varying picture quality. For the compression of video sequences, high-activity scenes will require more bits to achieve the same quality level as low-activity scenes. Also, within the same scene, some pictures will present more motion than others. Although more frequent, these fast-rate intra-scene variations present smaller fluctuations compared to the less frequent inter-scene variations. These time-varying requirements allied to the objective of efficient network usage calls upon the use of VBR channels. However, the use of VBR connections is not by itself an assurance that the requirements of the bit stream will be satisfied for the whole duration of the call together with an efficient usage of network bandwidth. Traffic parameters chosen at connection setup to closely match the initial characteristics of the video, may no longer be suitable after a scene change.

A dynamic UPC with adjustable renegotiable parameters is able to contribute to a more efficient use of bandwidth while meeting the requirements imposed by the low-frequency bit rate variations. Because the renegotiation process imposes a rather significant overhead to the network and because there is a latency in the response from the network, the renegotiation frequency should be kept as small as possible. It should only be triggered if the currently available bandwidth is not sufficient to guarantee the minimum target quality for all sources. Also, It should only be allowed at specified regular intervals. On the other hand, it should be controlled through the use of network feedback information. In this paper we propose to implement this control by using the explicit rate and congestion indication mechanisms specified in the ATM ABR service class.

To deal with the intra-scene variations we use the statistical multiplexer which aggregates a collection of VBR video sources and dynamically assigns bandwidth, in order to satisfy as fair as possible the level of quality indicated by each source. The objective is to obtain increased overall statistical gain while improving the quality of service for an aggregation of video sources.

\section{Motivation and Applicability}

Statistical multiplexing algorithms have been extensively studied and used to allow savings in overall transmission bandwidth in packet networks. Literature 
in this field is quite representative of the work already developed [2] ... 7]. More recently, researchers have started to study the potential gain, both in effective bandwidth and quality improvement, in the transmission of video signals over packet networks using adjustable UPC and traffic parameters renegotiation [8] ... [13. However the effects of using the two techniques combined have not yet been sufficiently explored. In [14], 15] and [16], the authors present approaches and results which demonstrate the benefits of multiplexing a set of video sources together with renegotiation of the traffic parameters of a single network connection for the aggregate stream. In our work we try to jointly explore the benefits of performing statistical multiplexing at the user level and dynamic adjustable UPC based on the following premises:

1. independent video sources are highly uncorrelated;

2. constant quality of compressed video is only possible with VBR;

3. constant quality compressed video presents two types of rate variability: lowamplitude/high-frequency intra-scene variations and high-amplitude/low-frequency inter-scene variations (respectively short-term and long-term in [2]);

4. renegotiation of traffic parameters provides a way of matching more closely the application requirements, but it imposes significant overhead to the network and consequent latency in the response of the network to those requests;

5. the same user can combine in a single ATM connection one or more video channels and share at his will the overall requested bandwidth while having to control one single network access unit;

6. a reduction in the number of renegotiation requests reduces the blocking probability of the network (or improves the network performance).

Of great importance is the existence of aplications which may actually benefit from the use of the proposed approaches.

We believe there are areas of potential application for the combined use of statistical multiplexing and dynamic renegotiation of traffic parameters, such as:

- in TV broadcasting, a supplier of TV programmes may use an ATM network as the backbone between a National Studio Centre and National terrestrial transmitters. It may hire a single channel and send through it 2 or more

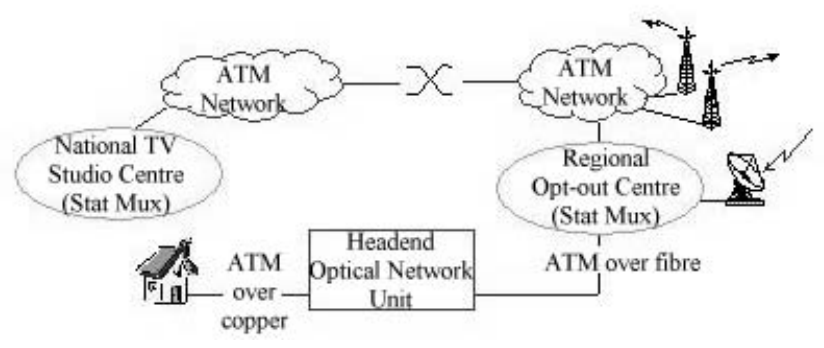

Fig. 1. Example of an application scenario 
TV programmes. The bandwidth it negotiates for with the network is dynamically shared among those programmes according to the user allocation bandwidth algorithm. The TV supplier has thus the freedom to choose and change at his own will the combination and number of simultaneous programmes and the distribution of bandwidth. Some work in this field was already developed in the ACTS project ATLANTIC [3];

- in TV studio production: journalists, editing producers, archivists siting at workstations interconnected by a local ATM network, access a/v material stored in networked distributed servers. Each user requests a single VBR connection and has a single connection manager runing locally in his machine. That single VBR channel is then shared dynamically by different video and audio sources. For example, a journalist when preparing the skeleton of his article will most certainly have to access a great number of different video and audio sources in a non-linear, non-sequential way. His local connection manager, comprising a statistical multiplexer and dynamic UPC manager will ensure that the required bandwidth to perform the desired operations will be available (if granted by the network) and shared in the best possible way. TV studio operations similar to these ones have already been experimentally implemented in the ACTS project ATLANTIC and in the framework of a Portuguese funded research project VIDION [4;

- in infotainment applications where users combine and interact with distinct video sources and other types of media, in a completely unstructured way;

- a supplier of VoD services, using an ATM backbone to interconnect remote islands of users to the central server where films are stored and accessed.

\section{Description of the Experiments}

\subsection{Overview}

The generic system concept is depicted in figure 2 All modules in our system implement closed-loop control algorithms, in the sense that they all react to information they receive from other modules.

The statistical multiplexer implements a dual closed-loop bandwith allocation algorithm which reacts to requests from the sources and to feedback information from the global dynamic UPC. Likewise, the UPC managers use a dual closed-loop parameters adjustment algorithm. The elementary UPC interacts with the real-time VBR video encoder and with the statistical multiplexer. The global UPC manager interacts with the network, receiving feedback information regarding network resources availability and with the statistical multiplexer.

Real-time VBR video encoders also use a closed-loop rate control mechanism to increase or decrease the rate of generation of bits. The rate adaptation is performed by modifying accordingly the values of the quantisation step sizes. Each real-time video source is VBR encoded and can be either an on-line (encoded on-line as it is being transmitted) or off-line (pre-encoded and stored material) video source. Our experiments have been restricted to the off-line case. 


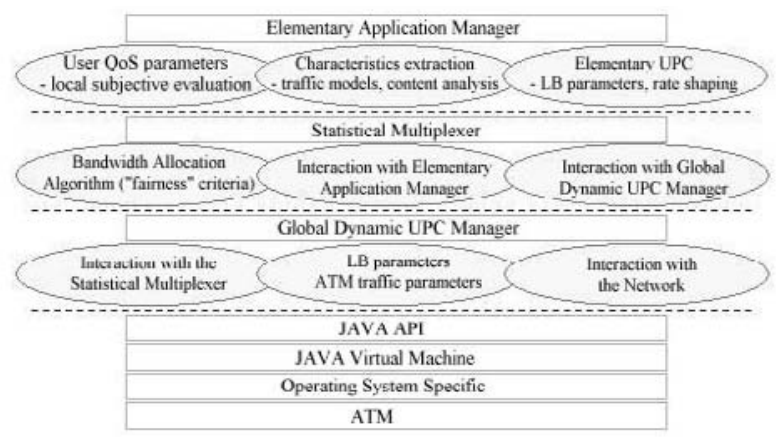

Fig. 2. Conceptual architecture of the system

Applications connect themselves to the system via their application manager, by suplying quality of service parameters from the user prespective: target picture quality (TPQ), minimum picture quality (MPQ) and service type (ST). With these parameters and a sample analysis of the bitstream to extract associated peak and mean bit rates, the application manager conveniently selects the parameters of the elementary UPC and passes the bit rate information and the user MPQ, TPQ and ST parameters to the statistical multiplexer. This entity estimates necessary values of peak and mean bit rates to allow the transmission of the aggregate traffic from all active sources. It passes this information to the user-to-network interface module, which then calculates the appropriate parameters of a global UPC capable of monitoring the aggregate traffic. It also specifies the ATM QoS and traffic parameters to request an ATM channel adequate to support the transmission of the multiplexed stream.

\subsection{Elementary Application Managers}

The application manager of off-line video sources extracts from the stored data, information concerning bit rates and service type. This information is directly used to select the parameters that better match the characteristics of the source. For on-line sources, there can be two alternative methods to obtain the same information: manually, through the intervention of a human, who supplies the desired user QoS parameters and bit rate information; automatically, with the application manager analysing a sample number of frames of the video source and using a parameterised set of values for the objective picture quality.

Once the desired user QoS and bit rates are specified, the parameters for a flow rate monitoring mechanism are calculated by the application manager. This momitoring is performed by an elementary dual Leaky Bucket mechanism the way as proposed by the ATM FORUM [1]. It comprises two LB, each one implemented through a finite-capacity buffer, the token buffer, with defined sizes and drain rates, $\mathrm{B}$ and $\mathrm{R}$, according to the desired peak and sustainable rates, length of bursts and spacing between bursts. One of the LBs monitors the peak 
rate with parameters $R_{p}$ and $B_{p}$ and, the other monitors the sustainable rate with parameters $R_{s}$ and $B_{s}$. The values of the LBs parameters are related with the traffic parameters in the following way:

$$
\begin{array}{ll}
R_{p}=P C R & B_{p}=1 \\
R_{s}=S C R & B_{s}=R_{p} * T-b \\
M B S=R_{p} / T & M B S=1+\operatorname{int}\left[\frac{\min \left(T_{1}-T_{s}, t_{s}\right)}{T_{s}-T}\right]
\end{array}
$$

$b$ : number of tokens drained from the token buffer $B_{s}$ in a frame period $\mathrm{T}$;

$T_{1}$ : minimum spacing that bursts of size equal to MBS must observe to be in conformance;

$t_{s}$ : burst tolerance.

The dual LB constraints the source such that its maximum and average bit rates do not exceed the negotiated PCR and SCR and that the length of bursts (number of consecutive cells transmitted with the minimum inter-cell spacing $\left.T_{p}=1 / R_{p}\right)$ does not exceed $M B S=\left(R_{p} \times B_{s}\right) /\left(R_{p}-R_{s}\right)$. The token buffers, initially set to their capacity $B_{p}$ or $B_{s}$, are continuously drained at a constant rate $R_{p}$ or $R_{s}$ and filled at the rate of submission of cells to the network. A cell may only be submitted to the network if it is able to add a token into the token buffer. If the buffer is full, the cell is not in conformance with the UPC and therefore should not be transmitted. The traffic parameters PCR, SCR and MBS are continuously evaluated for each GOP and their values set to:

$$
\begin{aligned}
P C R & =\text { bit rate of the largest picture in GOP } \\
S C R & =(1-k) \times R_{\text {avg }}+k \times P C R \quad \text { with } \quad 0<k<1 \\
R_{\text {avg }} & =\text { weighted sum of bit rates of all but the largest frame in the GOP }
\end{aligned}
$$

If significant changes are detected, the application manager requests a modification in the allocation of bandwidth to the statistical multiplexer.

We have performed experiments for stored video using accompanying metadata files with the following information: average and peak bit rates for each scene, initial and ending times of each scene (or the period during which the previous values are valid), frame period GOP structure and target picture quality. If this metadata is not available, the application manager must calculate them throughout the session lifetime or completely at connection set up.

\subsection{Statistical Multiplexer}

Statistical multiplexing, the ability of dynamically allocating bandwidth among sources according to picture activity and target quality, is based on the theory that independent video sources are likely to exhibit the same amount of activity simultaneously and therefore, the need for the same amount of bits.

Statistical multiplexing aims at providing better channel utilisation and better picture quality accross a number of video sources. The way it proposes to 
achieve this goal is to dynamically distribute the overall channel bandwidth, assigning variable amounts of bit rates to each source according to their present activity (complexity and motion). Statistical multiplexing has been traditionally applied to the use of a fixed-bandwidth channel by a group of VBR sources [17. and [18] and inside ATM networks to efficiently support a number of different connections (VCs) simultaneously [19] and 20].

To devise a methodology for the estimation of values for aggregate statistical bandwidth and UPC+traffic parameters, we have used traces of MPEG encoded video sequences. Compression video algorithms, such as those described in MPEG standards 2122], operate on a frame basis and use 3 different picture types: I (Intra), P (Predicted) and B (Bi-directional). Encoded pictures, belonging to one of the 3 possible types, succeed repeatedly in a structured way, the GOP structure. The amount of bits generated at the output of the encoder reflects this structure. Typically, within this GOP structure, I frames require more bits while $\mathrm{B}$ frames are those which require less bits.

The traces we have used are publicly available by Oliver Rose from the University of Wuerzburg ( ftp-info3.informatik.uni-wuerzburg.de/pub/MPEG). Each encoded sequence presents the following characteristics:

40000 frames at $25 \mathrm{~Hz}(T=40 \mathrm{~ms}) ; 384 \times 288$ spatial resolution; GOP structure with $N=12, M=3$;

$V B R$ encoded, fixed quantisation step sizes of 10, 14 and 18 respectively

for $I, P$ and $B$ frames.

Different transmission scenarios have been considered: sources transmitted separately both with a fixed set of UPC parameters for the whole stream and using renegotiation; the same, but using a GOP buffer to smooth the elementary bit rate prior to submit the stream to the network; association of different number of sources, considering both GOP-aligned and not-GOP-aligned and using a fixed set of UPC parameters; the same but using renegotiation of UPC parameters.

Table 1 presents the elementary sources behaviour in terms of mean and peak bit rates and degree of variablity in those bit rates. Statistics performed at the GOP level assume that each application manager has a buffer of sufficient capacity to be able to send all but the largest picture in the GOP at the SCR. The picture with the highest bit rate observed in the GOP is sent at that maximum rate, the $\mathrm{PCR}$. In table 1 , the average bit rate in $G O P$ is calculated using the bit rates of all but the largest picture in the GOP, while the max bit rate in GOP is set to the bit rate of the largest picture.

Table 2 characterises the behaviour of aggregate traffics in the same terms as for individual sources. It presents measures for combinations of different number of sources and assuming alignment or not at the GOP level. It also compares the bit rates required by those multiplexed bit streams with the bit rates required by the sum of corresponding individual sources, thus allowing the identification of statistical gain. It can be seen that using the same amount of rate variability as the threshold for initiating a renegotiation of the traffic parameters, the number of requests significantly decreases when 4 or more sources are multiplexed before accesing the network. When sources are not aligned at the GOP level, which we 
Table 1. Statistics of elementary sources

\begin{tabular}{|c|c|c|c|c|c|c|c|c|c|c|}
\hline \multirow[b]{4}{*}{ sources } & \multicolumn{4}{|c|}{ bit rate values (Mbps) } & \multicolumn{6}{|c|}{ number of variations in bit rate } \\
\hline & \multicolumn{2}{|c|}{ frame basis } & \multirow{2}{*}{\multicolumn{2}{|c|}{ GOP basis }} & \multirow{2}{*}{\multicolumn{2}{|c|}{ frame basis }} & \multicolumn{4}{|c|}{ GOP basis } \\
\hline & & & & & & & in avg & rate & in $\max$ & rate \\
\hline & avg & $\max$ & avg & avg of $\max$ & $25 \%$ & $50 \%$ & $25 \%$ & $50 \%$ & $25 \%$ & $50 \%$ \\
\hline$\overline{\text { asterix }}$ & 0.559 & 3.68 & 0.448 & 1.78 & 27042 & 18954 & 1049 & 380 & 338 & 104 \\
\hline $\operatorname{dino}$ & 0.327 & 2.00 & 0.232 & 1.38 & 25886 & 18496 & 770 & 244 & 179 & 42 \\
\hline simpsons & 0.588 & 3.52 & 0.487 & 1.70 & 27185 & 21238 & 683 & 219 & 275 & 69 \\
\hline lambs & 0.183 & 3.53 & 0.113 & 0.95 & 26058 & 18360 & 1050 & 411 & 179 & 47 \\
\hline movie & 0.357 & 4.31 & 0.259 & 1.44 & 28407 & 26970 & 1460 & 729 & 612 & 253 \\
\hline bond & 0.608 & 6.12 & 0.474 & 2.08 & 26980 & 26618 & 439 & 126 & 212 & 46 \\
\hline starwars & 0.233 & 3.12 & 0.154 & 1.10 & 26591 & 19930 & 1331 & 594 & 219 & 50 \\
\hline termin & 0.273 & 1.99 & 0.935 & 1.99 & 28663 & 23322 & 1321 & 466 & 463 & 92 \\
\hline$m T V-2$ & 0.615 & 5.73 & 0.512 & 1.75 & 28195 & 26429 & 1213 & 505 & 653 & 204 \\
\hline$m T V-1$ & 0.495 & 6.29 & 0.400 & 1.54 & 27207 & 20124 & 1198 & 504 & 596 & 213 \\
\hline race & 0.769 & 5.06 & 0.659 & 1.98 & 25335 & 16256 & 511 & 119 & 272 & 61 \\
\hline ATP-tour & 0.547 & 4.77 & 0.425 & 1.89 & 26827 & 19433 & 659 & 217 & 263 & 99 \\
\hline soccer & 0.678 & 4.68 & 0.560 & 1.98 & 27269 & 26752 & 427 & 126 & 273 & 86 \\
\hline sbowl & 0.588 & 3.52 & 0.487 & 1.70 & 27185 & 21238 & 683 & 219 & 275 & 69 \\
\hline
\end{tabular}

Table 2. Statistics of combined sources on a GOP period basis

\begin{tabular}{|c|c|c|c|c|c|c|c|c|c|}
\hline \multirow{3}{*}{\multicolumn{3}{|c|}{$\begin{array}{c}\text { Combination } \\
\text { of sources }\end{array}$}} & \multicolumn{3}{|c|}{ Average rate } & \multicolumn{4}{|c|}{ Maximum bit rate } \\
\hline & & & \multirow{2}{*}{$\begin{array}{c}\text { bit rate } \\
\text { value }\end{array}$} & \multicolumn{2}{|c|}{ variations } & \multirow{2}{*}{$\begin{array}{c}\text { avg of } \\
\text { peak rate }\end{array}$} & \multirow{2}{*}{$\begin{array}{c}\text { max of } \\
\text { peak rate }\end{array}$} & \multicolumn{2}{|c|}{ variations } \\
\hline & & & & $25 \%$ & $50 \%$ & & & $25 \%$ & $50 \%$ \\
\hline \multirow[t]{3}{*}{ all } & Sum & & 5.42 & 12794 & 4858 & 22.40 & 57.44 & 5001 & 1434 \\
\hline & $\operatorname{mux}$ & A & 5.24 & 22 & 1 & 22.87 & 30.97 & 0 & 0 \\
\hline & & NA & 6.56 & 8 & 1 & 8.35 & 14.35 & 29 & 3 \\
\hline \multirow[t]{3}{*}{12} & Sum & & 5.06 & 10142 & 3799 & 20.17 & 54.03 & 4127 & 1293 \\
\hline & $\operatorname{mux}$ & A & 5.06 & 31 & 1 & 20.17 & 27.99 & 3 & 1 \\
\hline & & NA & 5.95 & 20 & 1 & 7.641 & 13.66 & 108 & 7 \\
\hline \multirow[t]{3}{*}{8} & Sum & & 2.82 & 7838 & 3116 & 12.81 & 37.25 & 3032 & 1008 \\
\hline & $\operatorname{mux}$ & A & 2.82 & 247 & 17 & 12.81 & 19.252 & 23 & 1 \\
\hline & & NA & 3.54 & 86 & 3 & 5.345 & 11.104 & 144 & 29 \\
\hline \multirow[t]{3}{*}{6} & Sum & & 1.95 & 1846 & 2500 & 9.522 & 25.234 & 1846 & 614 \\
\hline & $\operatorname{mux}$ & $\mathrm{A}$ & 1.95 & 247 & 24 & 9.522 & 14.747 & 40 & 1 \\
\hline & & NA & 2.469 & 92 & 7 & 3.813 & 8.307 & 218 & 32 \\
\hline \multirow[t]{3}{*}{4} & Sum & & 1.587 & 3938 & 1570 & 7.130 & 1756 & 1392 & 1518 \\
\hline & $\operatorname{mux}$ & $\mathrm{A}$ & 1.587 & 279 & 28 & 7.130 & 11.287 & 62 & 3 \\
\hline & & NA & 1.927 & 145 & 15 & 3.295 & 6.934 & 165 & 20 \\
\hline \multirow[t]{3}{*}{2} & Sum & & 0.537 & 1819 & 624 & 3.863 & 9.8 & 517 & 146 \\
\hline & $\operatorname{mux}$ & $\mathrm{A}$ & 0.537 & 538 & 98 & 3.863 & 8.386 & 154 & 15 \\
\hline & & NA & 1.038 & 427 & 60 & 2.579 & 6.549 & 173 & 21 \\
\hline
\end{tabular}


understand as being the more frequent situation, larger gains in bandwidth are obtained. However, variability in peak rate is more pronounced when sources are aligned at the GOP level. Rate variability is the \% of change relative to the values in use. It can be noted that the inclusion of extra sources in the multiplex contributes on average with a reduction of $5 \%$ in the number of renegotiation requests. The values of rate variability (in \%) used to trigger the renegotiation are 25 and $50 \%$.

The estimation of bandwidth required for the multiplexed stream uses a simple algorithm that assumes sources are not aligned at the GOP level and that a reduction from $10 \%$ to $5 \%$ of the sum of bit rates is achieved for each new source joining the multiplex (see table 33). These values were obtained in the measures performed with the MPEG traces.

The statistical multiplexer continuously performs the bit rate calculations. If significant changes occur and on a $n-G O P$ basis (i. e., every $n * 12$ picture periods), it requests the dynamic UPC manager to alter the global UPC parameters. In that situation, the UPC manager may initiate with the network, negotiations for a new traffic contract (see subsection 3.4). After receiving a confirmation of the acceptance or rejection of the new traffic parameters, the UPC manager notifies the statistical multiplexer and adjusts its own policing parameters. If the new values were accepted, the statistical multiplexer distributes the bandwidth as requested. Otherwise it re-distributes the available bandwidth based on a fairness criteria, regarding values of bit rate requested and target quality level. If the bandwidth granted is not enough to completely satisfy all requests, the allocation algorithm starts by assuring that the minimum picture quality is maintained in all sources and then distributes the remaining bit rate according to a ratio between bandwidth requested and target picture quality.

Fairness allocation bandwidth algorithm. The statistical multiplexer uses a fairness allocation bandwidth algorithm based on the following definitions, measures and operations:

$$
L B_{\text {requested }}=\sum \max _{i}(1) \times(1-k)
$$

$\boldsymbol{L} \boldsymbol{B}_{\text {network }} \rightarrow$ bandwidth actually granted by the network $\boldsymbol{m a x}_{\boldsymbol{i}}(\mathbf{1}) \rightarrow$ maximum bit rate requested by source $\mathrm{i}$ to achieve the target picture quality level QoS(1)

$\boldsymbol{m a x}_{\boldsymbol{i}}(2) \rightarrow$ maximum bit rate requested by source $\mathrm{i}$ to achieve the minimum picture quality level $\operatorname{QoS}(2)$

$\boldsymbol{k} \rightarrow$ defines the multiplexing gain and depends on the the number of sources in the multiplex. Values for this parameter, obtained experimentally using the mentioned traces are given in table 3

Pseudo code

if $\left(L B_{\text {network }}>=L B_{\text {requested }}\right) \quad /^{*}$ assign bandwidth as requested for $\left(i=1 ; i<\right.$ numberOfSources; $\left.i^{++}\right)$ 


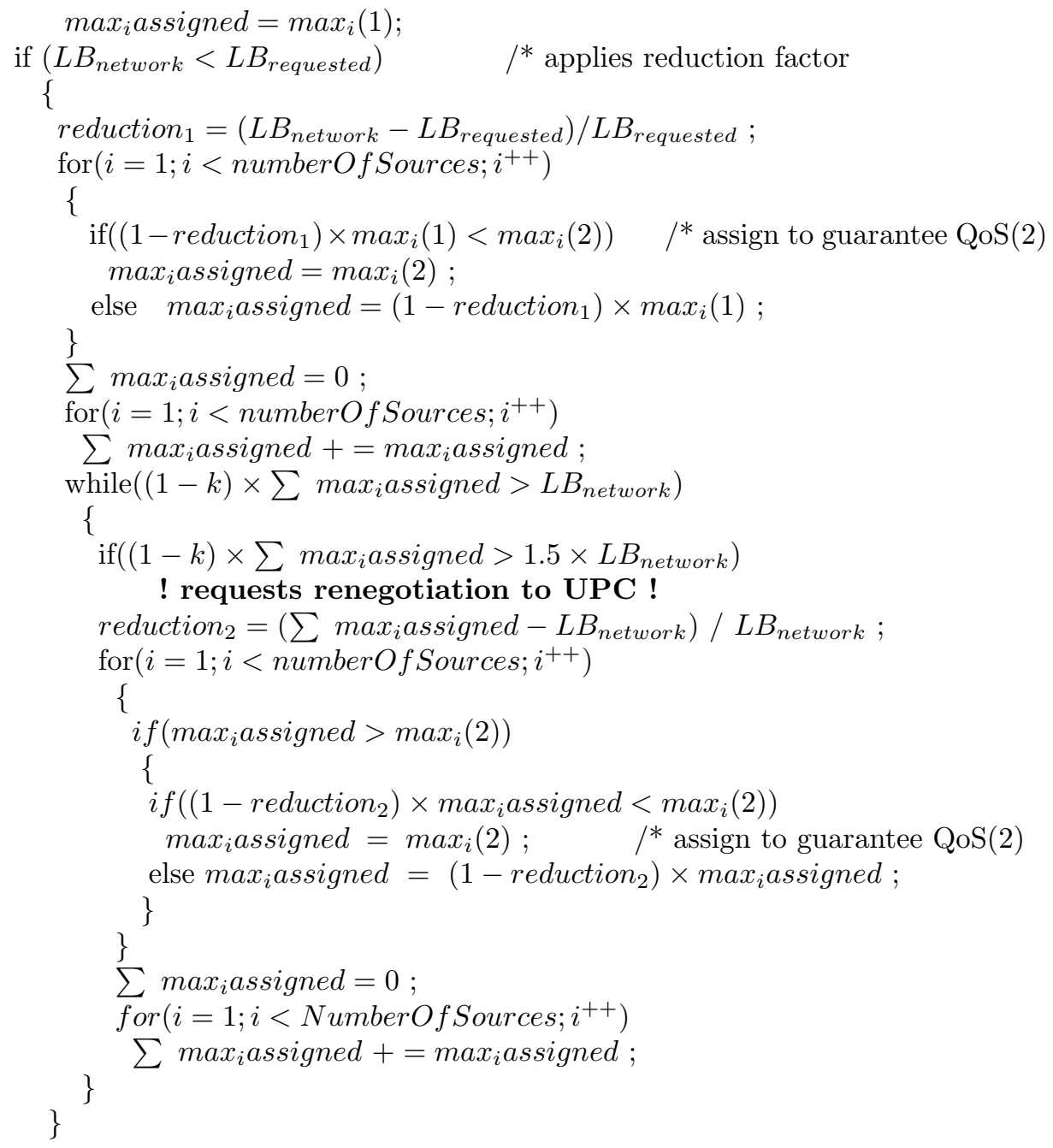

The set of calculations and operations are indicated for the distribution of the peak bit rate of the aggregate connection. The case we are considering, assumes that each elementary source, if transmitted isolated, would pass through a GOPbuffer before accessing the network and would have a peak rate set to the bit rate of the largest frame in the GOP.

\subsection{Dynamic Renegotiable UPC}

In ATM networks, the establishment of a new connection must be preceeded by the negotiation of a service contract between the new application and the network. The contract must identify the ATM service attributes requested from the network, through specification of traffic parameters (PCR, CDVT, SCR and MBS) and QoS parameters (peak-to-peak CDV, max CTD and CLR). 
Table 3. Experimental values for multiplexing gain

\begin{tabular}{cc}
\hline Number of sources & $k$ \\
\hline 2 & 0.30 \\
4 & 0.50 \\
6 & 0.60 \\
8 & 0.70 \\
12 & 0.75 \\
\hline
\end{tabular}

Once the connection is accepted, the network will provide the requested QoS for those cells that conform to the negotiated traffic values. If the user violates this traffic contract, the network no longer has the responsibility of maintaining the agreed QoS. It is therefore the interest of the user, to ensure that all cells submitted to the network do not exceed the negotiated values.

Conformance of the flow submitted to the network is monitored by an UPC mechanism implemented through a dual Leaky Bucket algorithm (see section 3.2). The global UPC manager sets its parameters according to the request sent by the statistical multiplexer. At connection set up, it negotiates with the network a traffic contract specifying the ATM traffic parameters PCR, SCR and MBS for the aggregate stream and selecting the specified QoS class 2. This specified QoS class supports a QoS that meets the performance requirements of the ATM service class suitable for real-time VBR video and audio applications [1]. Formulas for calculating the traffic parameters have already been presented in section 3.2

For each GOP period, consisting of 12 frame periods, the PCR is used during a frame period and the SCR during the rest of the GOP. This way there is a constant spacing of 11 frame periods between bursts. The values of the UPC are therefore calculated in order to match the requirements of the multiplexed stream as indicated by the statistical multiplexer. When a request for altering those parameters is received, the dynamic UPC before actually updating them, interrogates the network about the availability of resources.

Our approach to implement the interaction between the dynamic UPC and the ATM network is to use RM cells in a similar way as specified for the ABR service category [1]. The network access interface of the multiplexed source sends to the network RM cells with the indication of target bit rates. There are no values specified for the transmission frequency of those cells but typically, reported implementations send one RM cell for each group of 32 or 48 service cells. In response, the network sends feedback information in backward RM cells. This feedback consists of information about network bandwidth availability and state of congestion and can be explicitly stated in the fields Explicit Rate, Congestion Indication and No Increase of backward RM cells. The ER field of forward RM cells is used by the source to specify the target bit rate (the PCR). This value may be reduced by any network element while the cell is travelling from source to destination and again back to the source. Its final value when reaching the source dictates the highest rate that may be used by the source. RM cells can be 
of two types: in-rate or out-of-rate cells. The first type is included in the bit rate allocated to the connection, while the later is not. Out-of-rate forward RM cells are not taken into account to compute the source rate, but they may not be sent at a rate greater than 10 cells/s (the TCR, Tagged Cell Rate). As we propose to restrict the renegotiation frequency to once every $n \times$ GOP this rate of RM cells may prove to be acceptable. It is not possible however to report results in this field, namely of performance and feasibility of the proposed method and network blocking probability to renegotiation requests, because experiments have not yet been performed. It is however the intention of the authors to proceed the work in this direction.

\section{Results and Further Work}

We have evaluated our experimental system using traces of pre-encoded MPEG sequences. We have thus only implemented and off-line experiment of our proposed methodology, where the entire bitstreams to be submitted to the network were known in advance. Although having an argueably restricted field of application, this experience has revealed itself very important:

- it provided a way of evaluating the effectiveness of the proposed system:

- it provided valuable information to infere the methodology to use with

on-line cases, where video sequences are being real-time encoded as they

are being submitted to the ATM network.

We are developing an API in Java to provide applications the kind of functionality we have described for the elementary application managers combined with the dynamic renegotiation of UPC parameters. Presently, this API provides only basic functionality for native ATM applications to request an ATM connection and specify ATM QoS and traffic parameters.

In our premises at INESC, we have installed a $155 \mathrm{Mbit} / \mathrm{s}$ private ATM network with two FORE switches and a number of end stations equipped with 25 and $155 \mathrm{Mbit} / \mathrm{s}$ Fore ATM NICs. Using this infrastructure, we have set up a platform to test several TV studio applications developed within the framework of already mentioned research projects 3 and [4. The platform allows the experimentation of the application scenario TV studio pre and post-production, described in section 2. This scenario involves both pre-encoded material, thus the off-line case, as well as on-line real-time encoded sources. Our work will evolve towards the experimentation of the on-line case. For that matter we will use, once finalised, the full-featured API for native-ATM QoS-aware applications within the TV studio applications scenario. We will also produce experiments to further investigate the feasibility of using VBR/CBR service class combined with RM cells.

The results we have obtained so far can be extracted from tables 1 and 2 . They show us that it is possible to obtain statistical gain ranging from nearly $75 \%$ to $15 \%$ for the same picture quality objectives, depending on the number of sources being multiplexed. Also, that the frequency of renegotiation, initiated by 
the occurrence of the same amount of bit rate variation, is significantly smaller when sources access the network in a multiplex rather than individually.

\section{References}

1. ATM Forum Technical Committee: Traffic Management Spec Version 4.0 (1996)

2. N. Ohta, "Packet Video: Modeling and Signal Proc.", Artech House, Boston (1994)

3. AC078, ATLANTIC, Working documents, http://www.bbc.co.uk/atlantic

4. VIDION project, http://newton.inescn.pt/projects/vidion

5. O. Rose, "Statistical properties of MPEG video traffic and their impact on traffic modeling in ATM systems", Univ of Wurzburg, Inst of Computer Science (1995)

6. B. Maglaris et al., "Performance Models of Statistical Multiplexing in Packet Video Communications", IEEE JSAC, Vol. 36 (1988)

7. D. Heyman et al, "Statistical analysis and simulation study of video teleconferencing traffic in ATM networks", IEEE Trans. Circ. \& Sys. for Video, Vol. 2 (1992)

8. D. Reininger, Y. Senda and H. Harasaki, "VBR MPEG-2 Encoder for ATM networks with UPC renegotiation", NEC USA, C\&C Research Lab (1996)

9. D. Reininger, D. Raychaudhuri and J. Hui, "Bandwidth Renegotiation for VBR Video Over ATM Networks", IEEE JSAC, Vol. 14, No. 6 (August 1996)

10. D. Reininger, D. Raychaudhuri and R. Siracuse, "Video Transport in ATM networks: a systems view", NEC USA, C\&C Research Lab (1995)

11. B. Mark and G. Ramamurthy, "Joint source-channel control for real-time VBR over ATM via dynamic UPC renegotiation", NEC USA, C\&C Research Lab (1996)

12. B. Mark and G. Ramamurthy, "Real-time estimation and dynamic renegotiation of UPC parameters for arbitrary traffic sources in ATM networks", IEEE/ACM Transactions on Networking, Vol. 6, No. 6 (December 1998).

13. S. Giordano and J. Le Boudec, "The renegotiable variable bit rate service: characterisation and prototyping", EPFL ICA, Lausanne, Switzerland (1998)

14. E. Fulp and D. Reeves, "Dynamic bandwidth allocation techniques", North Carolina State Univ, Depts of ECE and CSC (1997)

15. Y. Nozawa et al, "Statistical multiplexing gateway for piecewise CBR transmission channel", Packet Video'99, New York USA (1999)

16. L. Teixeira and M. Andrade, "Joint Control of MPEG VBR video over ATM networks", ICIP 97, Sta Barbara, CA, USA (1997)

17. M. Perkins and D. Arnstein, "Statistical Multiplexing of MPEG2 video channels", SMPTE 136th Technical Conference, LA, USA, (1994)

18. Compression Labs white paper, "The next step in Digital Broadcast: statistical multiplexer enhances compressed video MPEG2"

19. G. de Veciana, G. Kesidis and J. Walrand, "Resource Management in wide area ATM networks using effective bandwidth, IEEE JSAC, Vol. 13 (1995)

20. K. Ross, V. Veque, "Analytic Models for Separable Statistical Multiplexing", Univ of Pennsylvania, Dept of Systems Engineering (1994)

21. Didier Le Gall, "MPEG: A video compression standard for multimedia applications", Transactions of ACM (1991)

22. ISO/IEC 13818-2, ITU-T Rec H.262, "Generic coding of moving pictures and associated audio: video", (1994) 